\title{
Improved 2D Digital Image Correlation Method for Displacement and Deflection Measurements of Structural Beams
}

\author{
Renee C. Oats ${ }^{1}$ and Qingli Dai, $\mathrm{PhD}^{2^{*}}$ \\ ${ }^{1}$ Ph.D. Graduate Researcher, Dept. of Civil \& Environmental Engineering, Michigan Technological University, \\ Houghton, MI United States \\ ${ }^{2}$ Associate Professor, Dept. of Civil \& Environmental Engineering, Michigan Technological University, Houghton, \\ MI United States \\ Email: qingdai@mtu.edu
}

\begin{abstract}
Digital imaging correlation (DIC) is an innovative approach for evaluating full-field mechanical deflections and time-dependent responses of structural members. An illustration of 2D DIC is shown for a rigid body translation of a small-scale steel beam. This paper presents improvements in deployment of 2D DIC for evaluating mechanical behaviors and responses of structural beam members. Improvement considerations regarding image resolution and speckle correlation design were emphasized for observing flexural behavior of steel and timber structural beams. A suggestive guideline for focal length configuration for observing displacements with 2D DIC and a contrasting speckle design emphasizing physical pixel sizing was presented. The DIC displacements were converted to physical scale measurements capturing within $1 \%$ error differences and subpixel accuracies when compared to traditional measuring devices and numerical modeling. DIC enabled detection of mid and full span deflection distribution in addition to time-dependent displacements advocating for DIC's versatility for structural beam evaluations.
\end{abstract}

Keywords: Digital image correlation, beam deflection, displacement distribution, finite element modeling, mechanical responses.

\section{Introduction}

It is critical to monitor the mechanical responses and deformation behaviour for structural beams under different loading conditions. These responses can indicate stresses, strains or displacement changes of infrastructure beam materials, which is imperative to understand for designing structural systems. Efficient assessment methods are demanded to evaluate condition in order to prevent varied beam materials and/or system failures. Generally, non-destructive monitoring devices such as Linear Variable Displacement Transducer's (LVDT), Ground Penetrating Radar (GPR) and strain gauges are deployed to measure deformation or displacement changes; however, these techniques can be costly, laborious and cumbersome to setup to observe not only maximum bending deflection measurements, but also initial fracture behaviour $[1,2]$. There is a need for automated inspection techniques to interrogate structural components over time-dependent processes, with submillimetre accuracies while also adhering to safety concerns $[3,4]$.

Digital Image Correlation (DIC) is an optical-based remote sensing technology in which digital images before and after loading are compared using automated computer algorithms in order to determine changes in position or deformation [5-7]. DIC has been regarded as a powerful technique in the field of experimental solid mechanics providing a low-cost approach for surface deformations, displacement field, strain and stress distributions [8-10]. Two-dimensional (2D) DIC involves simplistic image collection by deploying one camera for surface plane analysis as opposed to two cameras for integrated volumetric analysis regarded as three-dimensional (3D) DIC. This 2D DIC methodology is discussed through a small scale steel beam rigid body translation illustration. An improvement scheme in obtaining accurate DIC measurements when compared with traditional methods is presented. This paper incorporates DIC 
for measuring mid-span displacement and full-span deflection distributions of steel and timber structural beams. Numerical finite element modelling analysis also complements the experimental evaluation of the mid-span and full-span timber beam under cyclic time-dependent beam loadings. These results advocate that DIC as a comparable method for deformation and deflection evaluation of structural beams.

\section{DIC Technique and Accuracy Improvement}

\subsection{DIC Technique}

An illustration of the technique is shown in Fig. 1 in which a small scale $27.94 \mathrm{~cm}$ (11 in.) long, $10.16 \mathrm{~cm}$ (4 in.) wide steel $\mathrm{w}$ flange beam was subjected to rigid body translation with the intention that the $\mathrm{x}$ and $y$ displacements of the surface points are the same within this full field surface correlation. The beam was configured in a servo-hydraulic testing frame for a $0.125 \mathrm{~Hz}$ cyclic displacement testing at a peak displacement amplitude of $3.175 \mathrm{~mm}$ (0.125 in). A Canon EOS 7D camera with sensor size $3888 \mathrm{x}$ 2592 pixel resolution and a Canon EF lens of $50 \mathrm{~mm}$ (1.968 in.) focal length was positioned less than a 1 meter, $0.914 \mathrm{~m}$ (3 feet) away perpendicular to the steel beam. A light source was placed parallel to the camera illuminating the beam and a LVDT validated the beam displacement. A commercial software, Correlated Solutions', Vic2D was used to provide the DIC evaluation of the small steel beam collected images [11]. A speckle pattern, considered an integral part of the matching process, was created on the steel beam to enhance the pixel correlation. The optimal matching of a correlation pattern is determined by minimizing the criterion within the cross correlation coefficient criterion often dependent on the processing algorithm's specification $[7,9,12,13]$. Huang and Tsai [13] define the standard correlation function, C as:

$$
C(\Delta x, \Delta y)=\iint_{A} l_{0}(x, y) l_{i}(x+\Delta x, y+\Delta y) d x d y
$$

In this equation, $\mathrm{x}$ and $\mathrm{y}$ are the dimensions within the defined area of interest $\mathrm{A}$ whereas $l_{0}(x, y)$ and $l_{i}(x+\Delta x, y+\Delta y)$ are the gray-scale intensities of two or more images being compared [13]. In this rigid translation illustration, the region of interest, A was created along the entire user created painted speckle surface of the beam. Within the user-defined region of interest, a correlation grid or subset is created and a displacement vector is calculated for all the subsets. The conventional correlation calculation is carried out in steps along the pixel rows and columns of the grid region in each consecutive image for obtaining sub-pixel accuracy [9, 14]. Fig. 1 highlights the resultant translation displacement calculated during pixel correlation for the center of the beam of the gray-scale images. As this is assumed to be an entire plane translation, the intensity value for the displacement grid at the center is shown along the beam surface. The extremum coefficient correlation value was located and presented throughout along the surface from the p $(x, y)$ location to the deformed p' $(x+\Delta x, y+\Delta y)$ location in figs $1 \mathrm{a}$ and $1 \mathrm{~b}$.
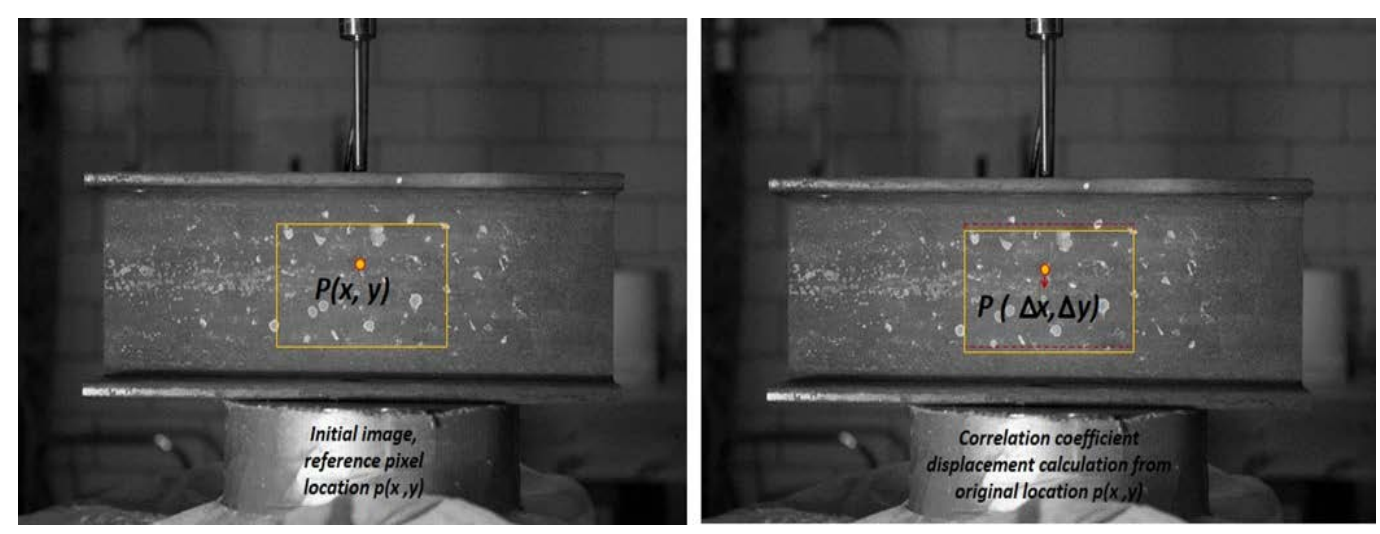

Figure 1. Image illustration of pixel location: (a) before and (b) after displacement along beam surface. 
DIC software detected image displacements in pixels and were converted to physical displacements (i.e. in millimetres) to compare with in-place traditional displacement sensor measurements. A percent error of the DIC measurement compared to the traditional method is presented as the relative error between the two measurements multiplied by $100 \%$. The DIC methodology revealed a percent error within $2 \%$ of the LVDT for the average displacement along the surface of the beam. As expected in rigid translation, the displacement was generally the same along the surface plane. Lens distortion (often unavoidable) is common to rigid body analysis, but also could be minimized with wider angle lens length $[15,16]$. The testing focal length was on the wider range of the lens scale at $50 \mathrm{~mm}$ to help demonstrate applicability of a low cost digital single lens reflex (DSLR) camera system.

\subsection{Measurement Accuracy Improvement}

In an effort to continue improving measurement accuracy, two parameters were identified for a closer examination: correlation in speckle design and position of the camera system regarding image resolution.

Pixel Speckle Pattern

For quality measurements, the subset correlation grids should contain enough speckle feature detail and appropriate resolution sizing to attain correlation values in the consecutive image given an anticipated displacement range. To explore the speckle size feature influence, four designs commonly used in DIC applications [17,18,19], including the previous surface painted speckle pattern (fig. 1), were deployed for rigid body translation observations for a small scale steel beam. Fig.2 (a-c) presents the remaining variations of speckle features (and pixel sizes) including a systematic marker (fig. 2a), predesigned speckle cover design (easily adjustable for scale) (fig. 2b), and natural surface texture (fig. 2c). These patterns present speckle designs encompassing larger scale field of view areas and wider speckle distribution designs than other researchers' investigations [20,21]. This research also evaluates physical sizing of speckles and placement of speckles coinciding with the image resolution consideration. For the minimal statistical sample pool, a random collection of 20 samples with $0.125 \mathrm{~Hz}$ cyclic testing rate for each of the four pattern categories is collected. Images collected were manually synched with the LVDT displacement measurements at a time increment of 1 second to intentionally sync sufficient data for the $3.175 \mathrm{~mm}$ displacement. Increase in data collection speed can be ideal for obtaining more images (i.e. data) depending on the loading frequencies or rates.

The Vic2D DIC processed results indicated the pre-designed highly contrasting pattern contained the smallest collective error of $1.14 \%$ (Table 1a). The painted speckle pattern shows the second greatest comparability capturing a $1.71 \%$ error with the strategic marker style and the natural surface pattern following comparison. The pre-designed pattern contained the most distinct speckle size variation with speckles sizing in the ranges of 2-61 pixels, surface painted pattern corresponding to 4-50 pixels, and the 15-40 speckles size range for the systematic marker pattern. The natural surface of the beam contained a small variation (under 5 pixels) speckle (deducing a small contrasting array of tracking pixels) which could correspond to the greatest error. Table 1 also presents an evaluation of the standard deviation of the 20 sample pool to consider how much the values vary from one another. The pre-design pattern incurred more intensity contrast and appropriate physical speckle size within necessary resolution. Again, these pattern investigations reflect considerations for in-field speckle pattern designs beyond artificial or simulated designs and for larger fields of views.

Image Resolution (Camera to Object Distance)

The resolution of the image typically describes how much detail or number of pixels appreciated in a digital image [22]. For exploration of the resolution and its relationship with the camera distance or specified focal length, the camera was moved to three different standing distances from the small scale steel beam, respectively $0.91 \mathrm{~m}, 1.22 \mathrm{~m}$ and $1.83 \mathrm{~m}$ (3, 4, and 6 feet). Crammond et al. (2010) reports magnifying optics considerations for smaller areas of interest such as $31 \times 31 \mathrm{~mm}$ or lower [21]. Images were captured at a $50 \mathrm{~mm}$ and then were increased to $100 \mathrm{~mm}$ focal length for the 4 and 6 foot positions which resulted in improved image quality. Further consideration incorporating theoretical optics theory revealed a suggested focal length for image collection with the relationship of standing distance multiplied by image size, divided by the sum of the specimen size and image size [23]. 


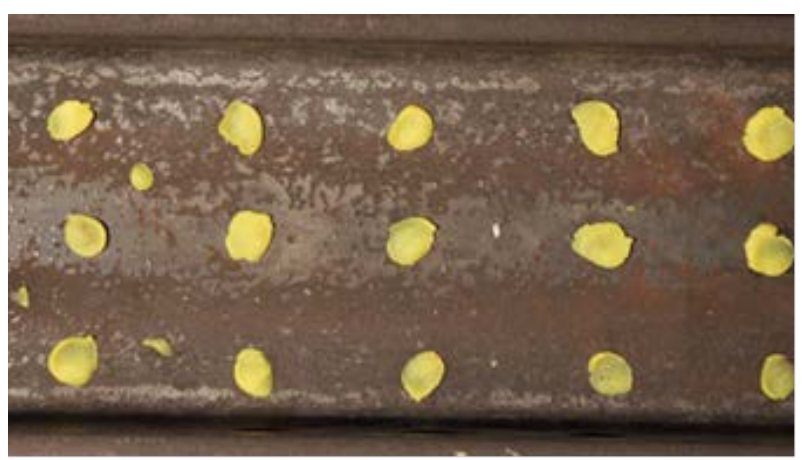

(a)

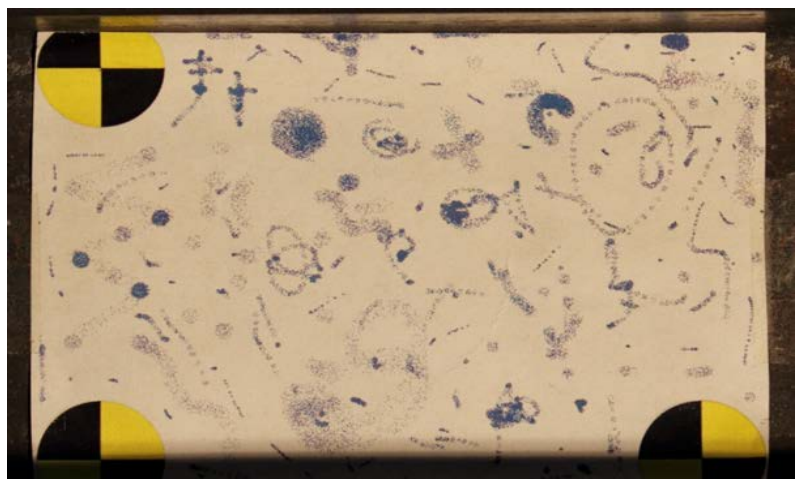

(b)

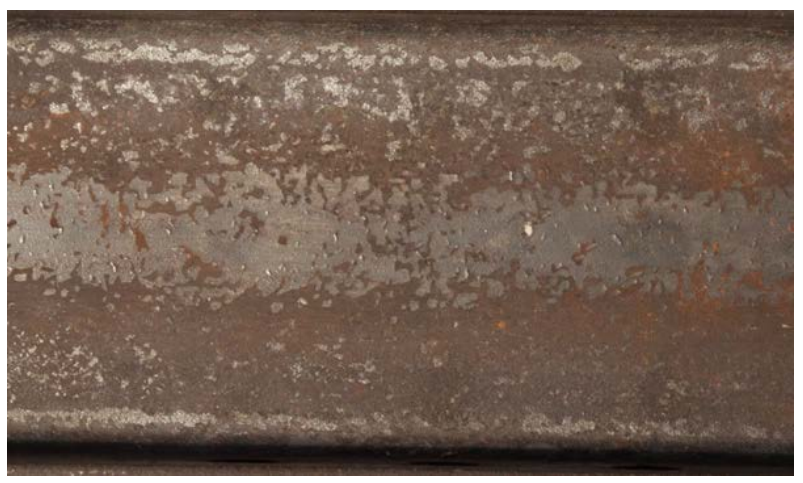

(c)

Figure 2. Small scale steel beam pattern alterations: (a) systematic marker pattern, (b) pre-designed speckle pattern and (c) natural surface feature pattern.

Table 1. Rigid body translation variation of (a) pattern speckle size variability and (b) standing distance \& focal length (resolution captured) influence.

(a) Pattern-Speckle Pixel Size Variations

\begin{tabular}{lccc}
\hline Pattern Type & Pixel Size Range (pixels) & \% Error & Std. Deviation (mm) \\
\hline Pre-designed speckle & $2-61$ & 1.144 & $8.31 \mathrm{e}-2$ \\
Surface painted speckle & $4-50$ & 1.708 & $2.14 \mathrm{e}-1$ \\
Systematic markers & $15-40$ & 2.711 & $1.24 \mathrm{e}-1$ \\
Natural surface markers & $<5$ & 3.849 & $2.33 \mathrm{e}-1$ \\
\hline
\end{tabular}


(b) Resolution-Focal Length/Standoff Position Variation

\begin{tabular}{lccc}
\hline Standoff Position/Focal Length & Image Resolution (mm/pix) & \% Error & Std. Deviation (mm) \\
\hline 4ft position w/ 90mm lens & $7.76 \mathrm{e}-2$ & 0.562 & $5.79 \mathrm{e}-2$ \\
6ft position w/ 135mm lens & $7.76 \mathrm{e}-2$ & 0.913 & $1.46 \mathrm{e}-1$ \\
3ft position w/50mm lens & $1.05 \mathrm{e}-1$ & 1.179 & $8.31 \mathrm{e}-2$ \\
3ft position w/25mm lens & $2.09 \mathrm{e}-1$ & 1.401 & $2.95 \mathrm{e}-1$ \\
$4 \mathrm{ft}$ position w/50mm lens & $1.39 \mathrm{e}-1$ & 2.091 & $2.95 \mathrm{e}-1$ \\
$6 \mathrm{ft}$ position w/50mm lens & $2.09 \mathrm{e}-1$ & 4.381 & $2.90 \mathrm{e}-1$ \\
\hline
\end{tabular}

For the 4 and 6 foot positions, given the $22.3 \mathrm{~mm}(.877 \mathrm{in})$ image sensor size and 11 in $(0.27 \mathrm{~m})$ beam size, the suggested focal length for obtaining quality lens capturing was $90 \mathrm{~mm}$ and $135 \mathrm{~mm}$. Therefore, the image resolution was determined as $7.76 \mathrm{e}-2 \mathrm{~mm} /$ pixel. Table $1 \mathrm{~b}$ lists the average percent errors of 20 cyclic testing sample pools for the 3,4 and 6 foot standoff position evaluations. The suggested focal lengths from the theoretical optics approach indicate a percent error below 1\% as shown for the 4 and 6 foot position. When the standing distance between the specimen and camera increases, the focal length and similarly field of view should be selected appropriately to effectively capture the motion at the lowest pixel resolution. In this case, the image resolution as the $7.76 \mathrm{e}-2 \mathrm{~mm} /$ pixel resolution is within the $3.175 \mathrm{~mm}$ rigid body displacement. A smaller field of view correlates with a smaller standing distance and a smaller resolution capturing more quantifiable data. Accordingly, the standard deviation increased with the pixel resolution highlighted in Table 1b. This focal length determination provides a suggestion or guideline to implement for capturing quality measurements, but is not inclusive to these exact values. The experimental illustration presents an improved 2D DIC method platform for measuring deformation and even characterizing mechanical behaviour (even at sub pixel levels). Mechanical testing of materials and structure stress analysis including full-field distributions are important and desirable [9], even for flexural bending beam observations, including time-dependent deflections.

\section{DIC Measurement of Beam Deflection and Displacement}

This section is to measure the displacements of varied linear elastic beam materials both on larger and smaller scales. Previous researchers incorporated artificial generated images to investigate flexural curvature for concrete materials $[24,25]$. In order to explore DIC's non-destructive ability to capture mechanical behavior and time-dependent deflection observation, a three-point bending test was performed on diverse structural members with the improvement strategies for magnification setup, specifically resolution and physical speckle size variability. A large scale steel beam and small scale timber beam were evaluated for maximum mid-span localized deflection and full-span total displacement evaluation.

\subsection{Large Scale Steel Beam Bending Test}

A $4.877 \mathrm{~m}$ (16-ft) W10x60 steel beam was loaded in a simply supported configuration using a100-kip self-reacting test frame. The steel beam was subjected to a $3.175 \mathrm{~mm}$ displacement control load at midspan with a load actuator frequency cycle of $0.1 \mathrm{hz}$. Based on the accuracy improvement considerations, the suggested focal length was actually determined as $45 \mathrm{~mm}$ from the resolution relationship of the image size $(22.3 \mathrm{~mm})$, distance of camera to steel beam, $0.609 \mathrm{~m},(2 \mathrm{ft}$ ) and the $27.94 \mathrm{~cm}(11 \mathrm{in}$.) size of interest area on the steel beam's surface. An LVDT is positioned under the bottom of the flange midspan to corroborate with the DIC measurements. A speckle pattern was created with two different spray-painted layers to provide a unique density size on the surface. Fig. 3a provides a close up of the testing setup with the image collecting camera. A light source was placed parallel to the camera to provide illumination on the beam's speckle pattern. Changes in the speckle pattern design and focal length were also altered to promote the improvement approach discussed. 


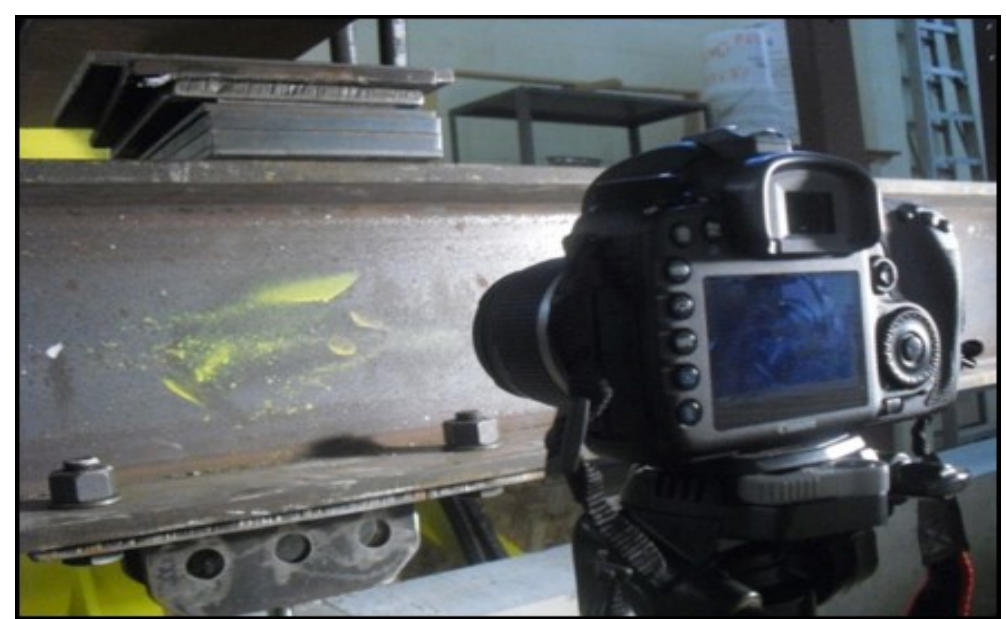

(a)

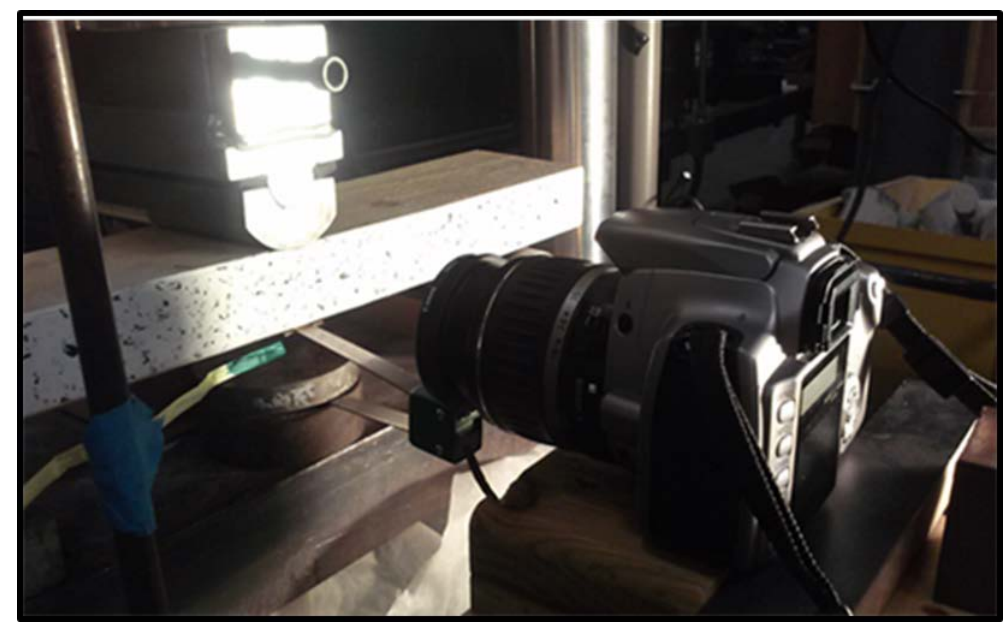

(b)

Figure 3. Beam bending test cases: (a) large scale steel beam with a LVDT underneath bottom of flange and (b) small scale timber beam with attached gauge.

\subsection{Small Scale Timber Beam Bending Test}

For observation of mid-span deflection and full-span displacement distribution, a $3.8 \times 8.9 \times 45.7 \mathrm{~cm}$ (1.5x3.5x18in.) timber beam was configured in an inverse simply supported configuration in a Materials Testing System (MTS). Therefore, the actual load was guided from the bottom supports positioned along the horizontal length of the beam at $7.3 \mathrm{~cm}(2.875 \mathrm{in}$.) and $38.4 \mathrm{~cm}$ (15.125in.). The tandem 55-kip structural actuators testing system contained a cyclic frequency of $0.125 \mathrm{~Hz}$ at a near1and $2 \mathrm{~mm}$ displacement controlled loading for the timber beam (shown in fig.3b). A Nikon 5100D camera captured images at a resolution of 3456 x 2304 pixels. A $55 \mathrm{~mm}$ focal length was suggested from the discussed relationship incorporating the image size $(23.6 \mathrm{~mm})$, the original camera to steel beam distance for midspan emphasis $(21.59 \mathrm{~mm})$ and the size of area of interest equal to $6.985 \mathrm{~cm}$ as the area underneath the load head. After mid-span deflection observations, the camera distance was tripled near 66cm to capture as much of the beam at the $55 \mathrm{~mm}$ focal length for full span observation. A similar light source was placed parallel to the camera to enhance the speckle pattern comprised of an array of pixel sizes range (1-55 pixels per speckle). For this evaluation, an open source DIC MATLAB algorithm using the image processing toolbox provided a calculation of the full field displacement and strains from a set of images. Accuracy for this DIC MATLAB algorithm was generally defined within 0.1 pixel [26]. 


\section{Results of DIC Analysis Results of the Large Scale Beam}

\subsection{Mid-span Beam Deflection Measurement between DIC and LVDT}

The $7.7 \mathrm{e}-2 \mathrm{~mm} /$ pixel image resolution with this $45 \mathrm{~mm}$ focal length is within the $3.175 \mathrm{~mm}$ anticipated deflection. Fig. 4a provides an illustration of this correlation function calculation in the processed DIC displacement result. In this image, the pixel intensity location along the bottom of the beam highlighted in the processed area was compared to the LVDT positioned at the similar location from the ground. The color contour illustrates higher displacement at the top of the beam where the concentrated force is applied. The DIC displacement contour commonly reveals changes in pixel scale; however, the displacement contour in a physical measurement scale calculated in the Vic2D processing software is presented.

Fig. 4a reveals a $3.14 \mathrm{~mm}$ (0.1237in.) displacement as it is very close to the anticipated $3.175 \mathrm{~mm}$ value. This DIC value was compared with the LVDT measurements and a deflection response for one bending cycle between these two values is illustrated (fig. $4 \mathrm{~b}$ ). This comparison reveals a $0.6 \%$ percent error for evaluating linear elastic curvature behavior. Additionally, this resolution was within the speckle size range, which represented painted speckles as small as 0.5 pixel.

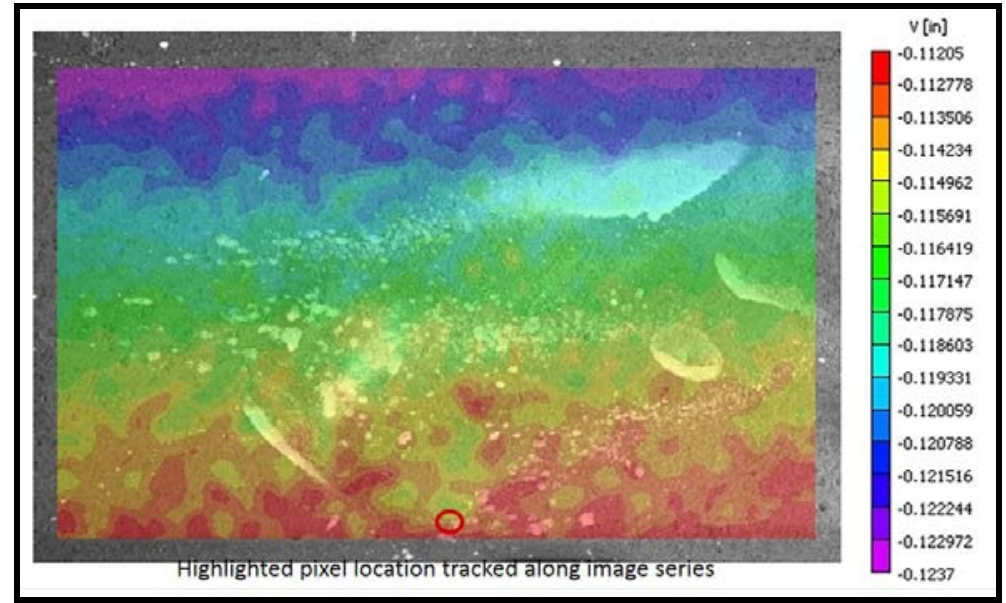

(a)

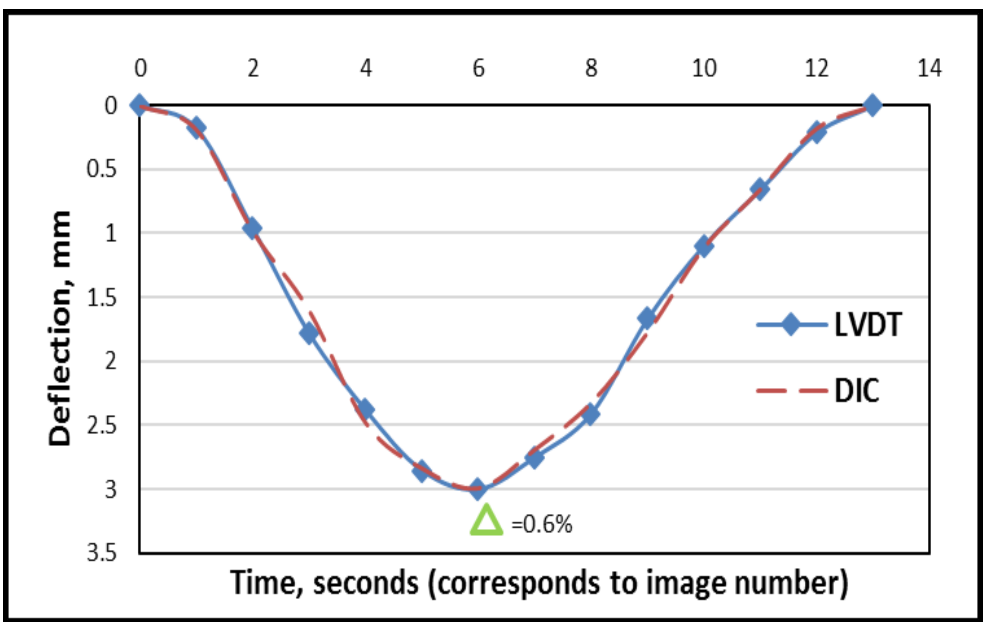

(b)

Figure 4. Large scale steel beam results: (a) processed image of deformed DIC correlation results and (b) graphical comparison of DIC and LVDT deflection measurements. 


\subsection{Mid-span Deflection Measurements Affected by Setup Parameters}

To illustrate the influence of standing distance resolution and efficient speckle features, two additional test series are presented for observing 0.125 " deflections. The $45 \mathrm{~mm}$ focal length was altered to a $25 \mathrm{~mm}$ and $50 \mathrm{~mm}$ focal length at a slightly larger camera distance, 2.75 feet $(83.82 \mathrm{~cm})$ considering alterations in accuracy. Table 2a presents sample results of this observation for each of the unique positions. The percent errors between the LVDT and DIC generally increase as the resolution increases as miscorrelation of sufficient data may occur.

Table 2. Flexural bending data under factor variation of (a) standing distance \& focal length variation (resolution captured) and (b) pattern speckle size variability.

(a) Resolution -Focal Length/Standoff Position Variation

\begin{tabular}{lcc}
\hline Standoff Position/Focal Length & Image Resolution (mm/pix) & \% Error \\
\hline 2ft position w/45mm lens & $7.77 \mathrm{e}-2$ & 0.602 \\
$2.75 \mathrm{ft}$ position w/ 50mm lens & $9.62 \mathrm{e}-1$ & 1.224 \\
$2.75 \mathrm{ft}$ position w/ 25mm lens & $1.92 \mathrm{e}-1$ & 2.834 \\
\hline
\end{tabular}

(b) Pattern-Speckle Pixel Size Variations

\begin{tabular}{lcc}
\hline Pattern Type & Pixel Size Range (pix) & \% Error \\
\hline Surface painted speckle & $2-14$ & 1.224 \\
Systematic markers & $4-20$ & 1.063 \\
Natural surface features & $<5$ & 1.607 \\
\hline
\end{tabular}

Table $2 \mathrm{~b}$ also presents the evaluation of two additional design patterns, a strategic marker design and a natural surface feature correlation under the previous $50 \mathrm{~mm}$ focal length. The marker design percent error was the smallest as the bottom of the beam location compared to the LVDT contained greater pixel contrast for optimal correlation. It should be noted additional factors beyond those in Table 2 such as lighting or even the perpendicular position of the camera can align closely with the pattern and distance alignment accuracy influence concerns. Additional information on this exploration of additional setup parameters and negating strategies for accurate testing measures are presented elsewhere (Oats et al., in review, 2017).

\section{Results of DIC Analysis Results of the Small Scale Beam}

\subsection{Mid-span Beam Deflection between DIC and Displacement Gauge}

The timber beam was setup in a three-point bending configuration in the inverse direction with the MTS load head being the confined static locale and the loading guided from the bottom supports. The mid-span beam deflection was determined by subtracting the displacement at the center (location B) from the support location (location A) to present a true deflection value comparative of the full beam displacement contour shown in Fig. 5a. The support locations reveal a slightly higher motion as support locations were the loading forces in this setup. The image resolution at this position was 7.2e-2 $\mathrm{mm} /$ pixel enabling more details during image correlation. Fig. 5b illustrates the graphical presentation of the total deflection between the gauge and the DIC image processing for a flexural bending demonstration of 1 and $2 \mathrm{~mm}$. Displacements of $1 \mathrm{~mm}$ and $2 \mathrm{~mm}$ were expected; however, the synching displacements were captured slightly shy of $1 \mathrm{~mm}$ at $0.914 \mathrm{~mm}$ and $1.5 \mathrm{~mm}$ for $2 \mathrm{~mm}$. The DIC and LVDT mid-span percent errors for the $1 \mathrm{~mm}$ displacement peaks were $1.15 \%$ and $2.46 \%$, respectively. Percent errors were slightly larger for the $2 \mathrm{~mm}$ peak correlations over $2 \%$. Even with slight fluctuation (minor noise) throughout the continuous flexural cycle, the graphical plots present similar behavior observations between DIC and traditional devices. These results support DIC can observe sub-millimeter displacements relative to under 1 pixel scales detailing validity of method for larger or small scale deformations of materials. 


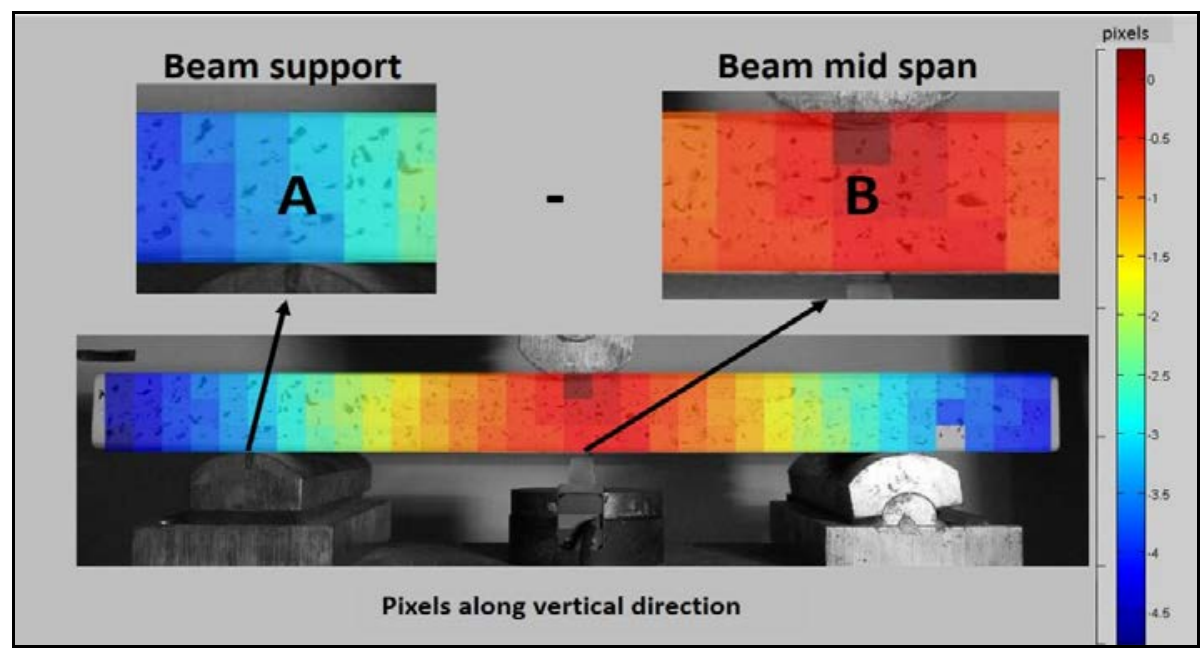

(a)

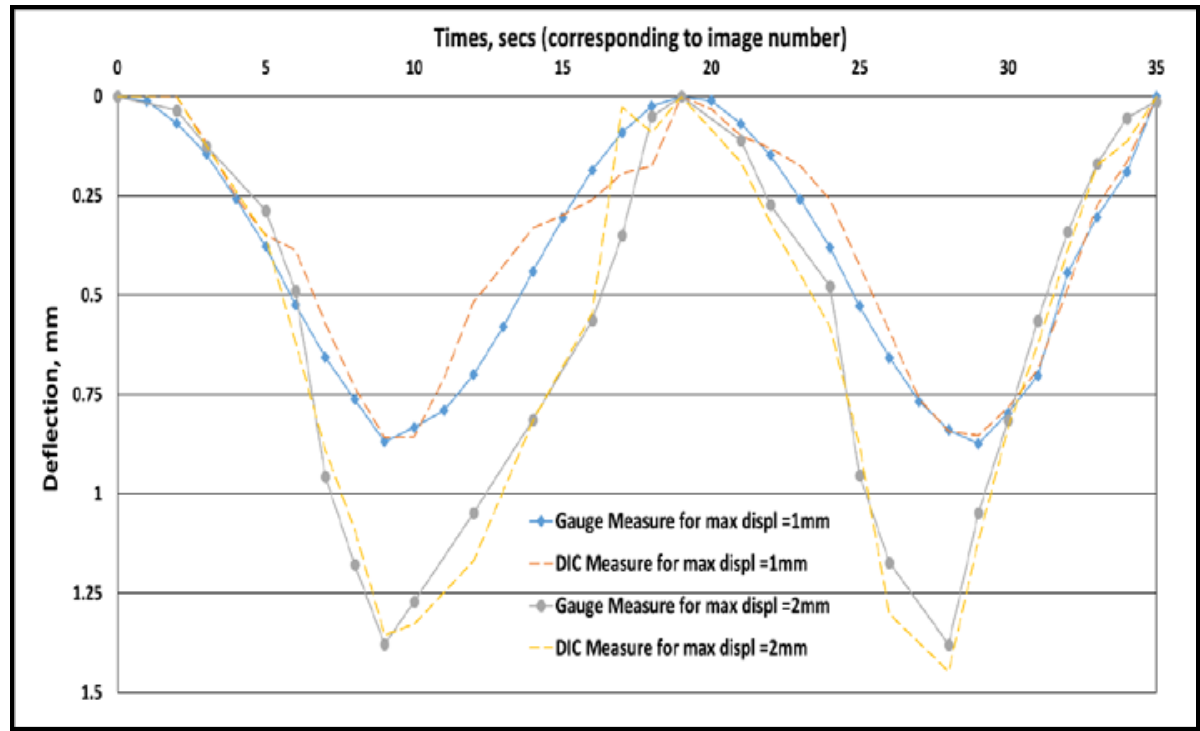

(b)

Figure 5. Timber beam results: (a) DIC results with highlighted mid-span location B and support location A and (b) graphical comparison of DIC and displacement gauge mid-span deflection measurements.

\subsection{Mid-span Beam Deflection between DIC and FEA}

An ANSYS finite element analysis (FEA) numerical model was created to compare with the DIC displacement distribution along the structural beam under loading (fig.6a). For simpler computation, half of the 18-inch beam was incorporated as a symmetric beam model for numerical analysis. A 20-node solid186 element was used for model inputs along with the beam's size dimensions and properties including Young's Modulus value of 2.3e6psi, Poisson's ratio of 0.3 , and a density value of $1.79 \mathrm{e}-2$. To simulate the boundary condition and the inverse three-point bending scheme, there was a $1 \mathrm{~mm}$ displacement controlled load at the 2.875in. support location with the confined zero displacement at the far right of beam spreading across a $19.05 \mathrm{~mm}$ (0.75in.) width. Fig.6a illustrates the simulated load from the support location as well as the displacement contour with the smallest displacement as the blue shade and greatest at the free end of the beam in red. The displacement at the center of the beam was $0.043 \mathrm{~mm}$ (0.170e-2in.) whereas the displacement at the support location was $0.9144 \mathrm{~mm}(0.036 \mathrm{in}$.) in 
which the difference for the mid-span location would be $0.8722 \mathrm{~mm}(0.0343 \mathrm{in}$.). The DIC value at this location is $0.8585 \mathrm{~mm}$ (0.0338in.) for the initial load peak, producing a $1.45 \%$ percent error.

In addition, the comparison between FEA and DIC was further conducted by examining the vertical displacement distribution along the $\mathrm{x}$ direction of the beam. Two different images captured within a 2 second time period were compared between DIC and FEA as shown in fig. 6b. The results shown here follow the half beam description from fig.6a with the end of the beam on the left and panning right to the mid span of the beam along the horizontal direction. For both of the time steps, FEA aligns well with this DIC distribution for majority of the beam's surface, with slight dissention particularly, as the beam nears to the mid-span. Slight variability is found as the FEA approaches the mid-span in which the boundary and bending configurations in the experimental setup and model differs slightly. The FEA and DIC resembles similar displacement distribution behavior supporting DIC could be advantageous for disclosing beam behavior trends.

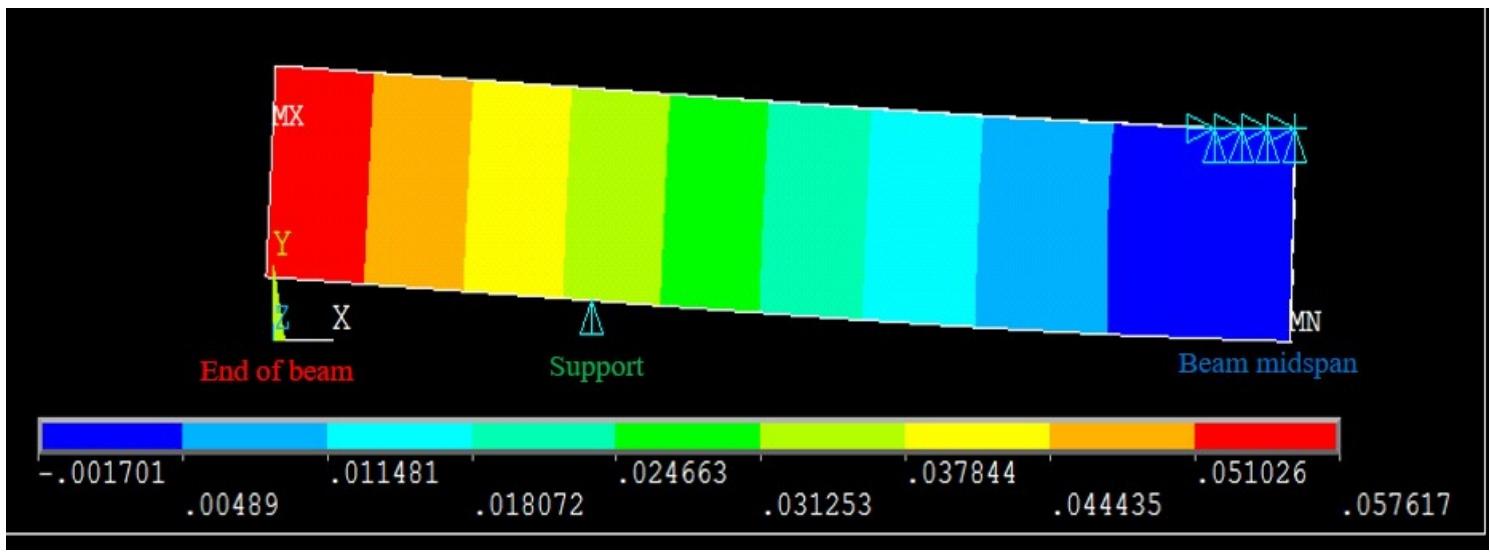

(a)

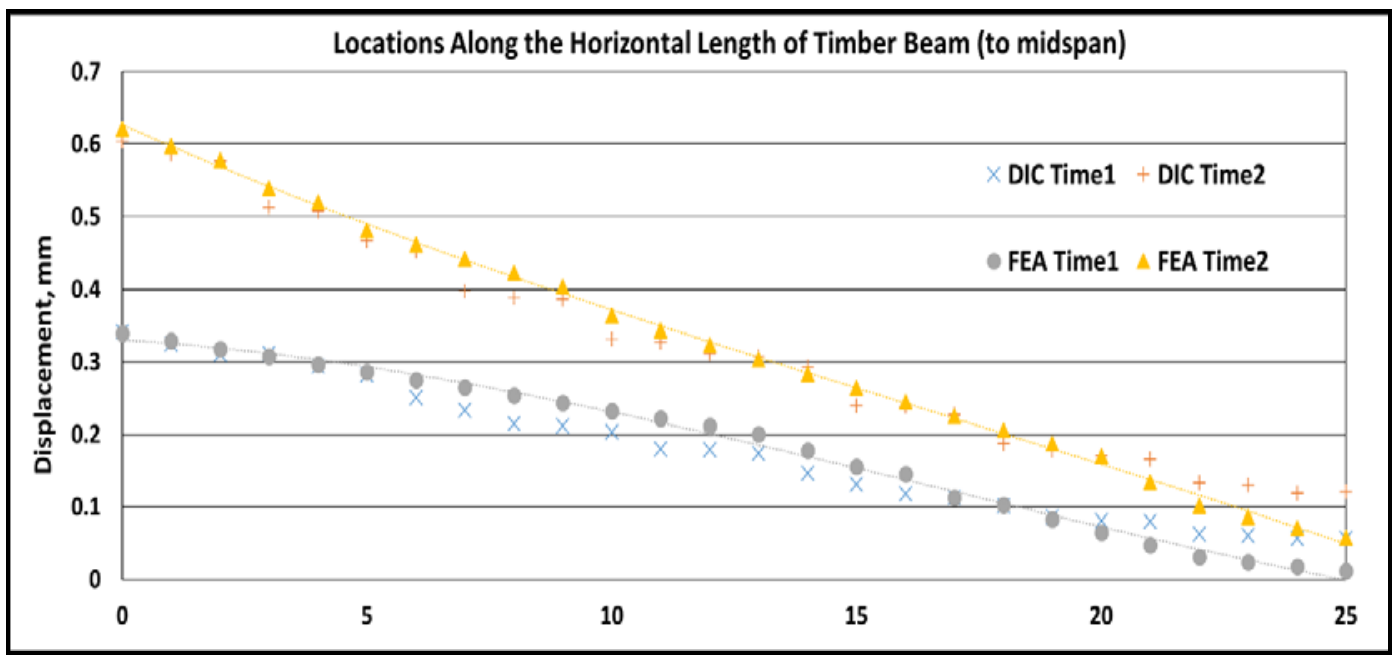

(b)

Figure 6. Timber beam FEA results: (a) FEA Model of symmetric half of timber beam under loading and (b) FEA and DIC vertical displacement distribution comparison along the symmetric horizontal beam length.

\subsection{Full Span Beam and Time-Dependent Displacement between DIC and FEA}

An evaluation for the full timber beam span under the $0.914 \mathrm{~mm}$ flexural bending was captured and compared with FEA. Figs. $7 \mathrm{a}$ and $7 \mathrm{~b}$ present four images including the initial position and corresponding pixel contour outputs for the timber beam under loading. Fig. 7a presents the scale contours along the full horizontal length of the beams throughout the loading cycle. The greatest 
movement is near those support locations (at the ends) with the smallest at the static load head. Fig. 7b shows the graphical representation along the surface of the beam horizontal (x-direction) length in pixels. This figure presents the length in pixel subsets along the centralized beam length. There is also a noticed missing correlation subset near the right support shown on both results outputs highlighting imposed noise or variability in DIC correlation measurements; however, the method is still able to provide a detailed observation along the bulk of the beam's surface.

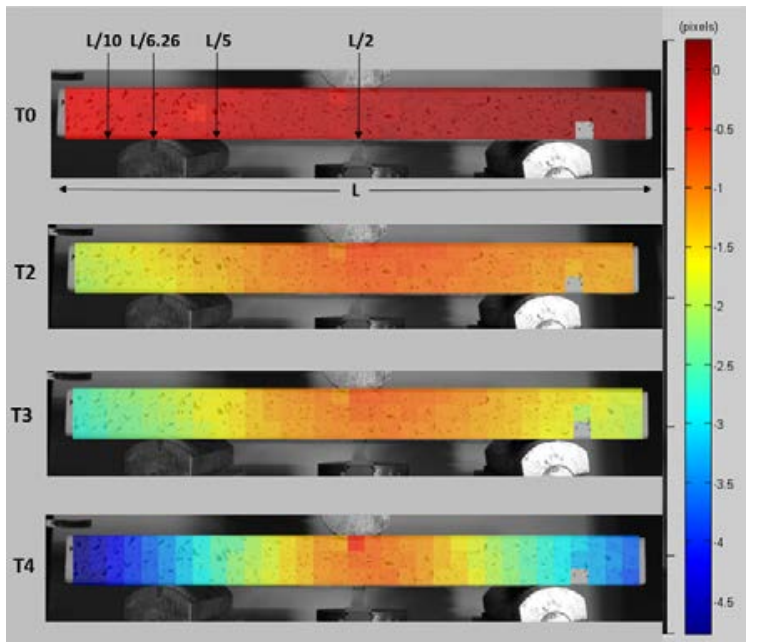

(a)

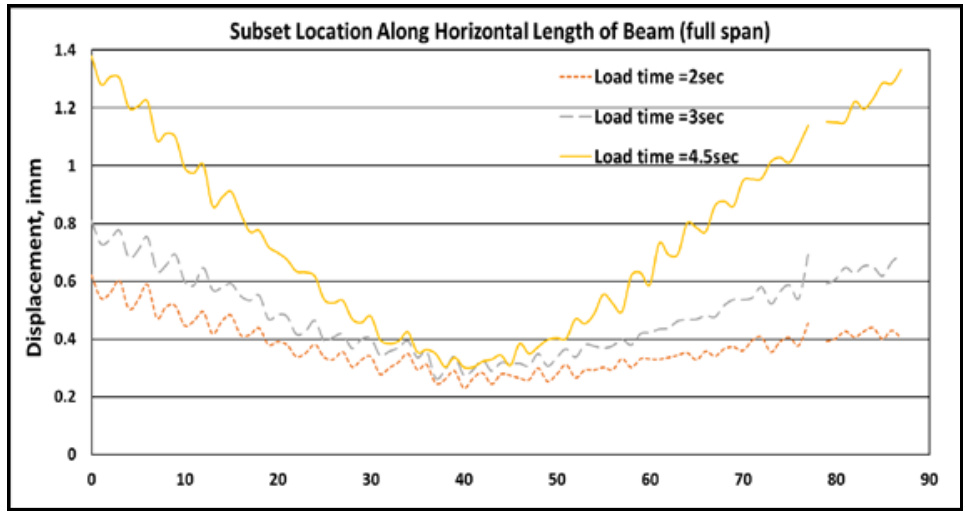

(b)

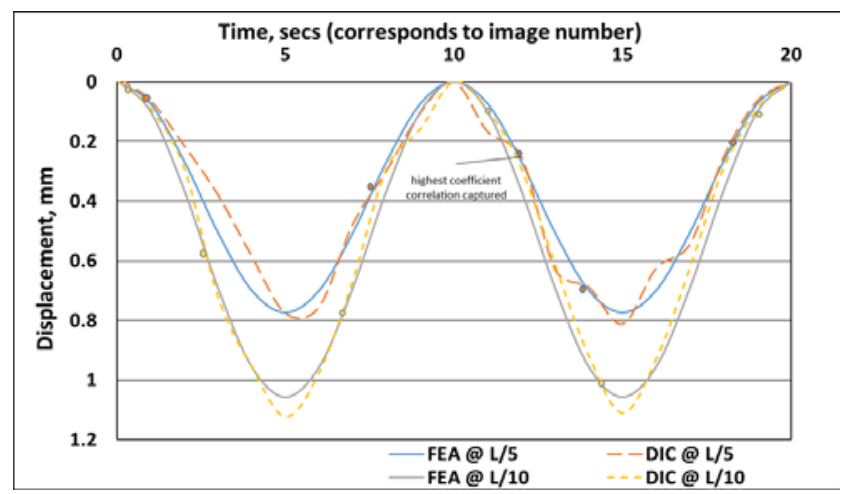

(c)

Figure 7. Timber beam DIC and FEA measurements: (a) full span DIC deflection contour images over time, (b) DIC displacement distribution along horizontal neutral axis of beam and (c) FEA and DIC time-dependent displacement responses at both L/5and L/10 locations. 
As the displacement of the beam's mid-span location is disclosed only with the LVDT, the displacement measurements at other locations can be detailed with DIC and correlated with FEA results. For example, the location L/3, (equivalent to 6in.) displacement along with other locations in numerical modeling can be revealed from fig. 7 (collected at T3 image). The node is tracked in FEA under the flexural bending and reveals a physical measurement of $0.408 \mathrm{~mm}$ corresponding to the orange colored contour location presented in a pixel scale. For the DIC evaluation within this L/3 location, a 1.163 pixel displacement was determined and multiplied with $3.5 \mathrm{e}-1 \mathrm{~mm} /$ pixel resolution factor (based on image size specifications) revealing a $0.4076 \mathrm{~mm}$ displacement. The FEA result (at T3 image time series) revealed a $0.4084 \mathrm{~mm}$ displacement and resultantly a $0.1869 \%$ error indicating accurate measures between the two methods. Correspondingly, several beam locations, specifically L/5, L/6.26, L/8 and $\mathrm{L} / 15$, equivalent to $9.144 \mathrm{~cm}$ (3.6in.), $7.073 \mathrm{~cm}(2.785 \mathrm{in}),. 5.715 \mathrm{~cm}(2.25 \mathrm{in}$.) and $3.048 \mathrm{~cm}$ (1.2in.) are highlighted in Table 3 (a few are shown on fig. 7a) to help illustrate the average error between the FEA and DIC measurements along the beam's surface. Correspondingly, the average error over the whole beam surface (this case representing the symmetric beam half) was slightly over $1 \%$ error.

Comparison of time-dependent measurements with in-contacting devices was shown in section 5.1, but can also be illustrated with FEA under continuous cyclic loads. Two of the FEA model node locations, $\mathrm{L} / 5$ (presented in Table 3) as well as a bonus location L/10 (equivalent to $4.572 \mathrm{~cm}$ ) were selected to promote the applicability of this technique for providing measurements along the timber beam's length over time. FEA modeling for the timber beam was performed under harmonic transient cosine curvature analysis over a two 10-second timed cycles with a displacement controlled $0.914 \mathrm{~mm}$ loading at the 2.875 in location along the x-direction of the beam. Fig. 7c reveals a comparative FEA time-dependent post processed curvature response for the harmonic modeling at the $\mathrm{L} / 5 \mathrm{and} \mathrm{L} / 10$ node locations resemble the experimental DIC curvature response with great correspondence. With this demonstration, DIC is able to provide full-field displacement evaluation along the beam in which other methods may be limited in capturing structural changes at specific locations and over time.

Table 3. Percent error comparison of FEA and DIC displacement measurements

\begin{tabular}{llll}
\hline Location & FEA (mm) & DIC (mm) & \%Error \\
\hline $\mathrm{L} / 3$ & 0.4084 & 0.4076 & 0.1869 \\
$\mathrm{~L} / 5$ & 0.6959 & 0.6883 & 1.1070 \\
$\mathrm{~L} / 6.26$ & 0.9144 & 0.9398 & 2.7777 \\
$\mathrm{~L} / 8$ & 1.1176 & 1.1226 & 0.4525 \\
$\mathrm{~L} / 15$ & 1.3258 & 1.3396 & 1.0306 \\
\hline
\end{tabular}

Average \% Error $=1.0960$

These case studies illustrate condition analysis of structural beam components, which can be used to validate finite element models and provide characterization of material properties. As the FEA exploration illustrates, DIC closely resembles displacement measurement from models and can reflect stress-strain responses or experimental design analysis for infrastructure beams. DIC is capable of providing an evaluation of various ranges of motion (rigid translations or bending behavior) and timedependent observations. The recommendations provided improved the accuracy of 2D DIC measurement even under $1 \%$ error difference or 0.5 pixel, but care should be given as accuracy is not limited to these factors alone. In addition, execution of sufficiently timed data collection and acquisition can promote true accurate data comparisons.

\section{Concluding Remarks}

In this study, an improved 2D-DIC technique approach was deployed to evaluate mechanical behavior for steel and timber structural beams. DIC accuracy improved when considering relationships for camera parameters such as image resolution corresponding to standing distance and unique speckle pixel size variation during image collection. Understanding and adhering to best strategies for incorporating DIC for deflection structural beam evaluation presents a small error even under $1 \%$ error difference when compared to traditional techniques. Mechanical evaluations for structural beams were presented highlighting DIC's flexibility capability for capturing accurate deflections under localized mid-span and 
full span three-point bending testing. DIC compares largely with numerical modeling and traditional devices for static and time-dependent loading interactions regardless of algorithm design. As a part of this study, the following conclusions can be gathered:

$>$ DIC accuracy improvements adhering to image resolution, adequate speckle contrast distinction and size variability as noted in section 2 can be achieved

$>$ DIC captures sub pixel localized displacement and full field deflection changes on structural beams highlighted in sections 3 and 4 and 5

$>$ DIC enables time-dependent and comparable material characterization for beams shown in sections 4 and 5

DIC displacement or deflection measurements can assist in mechanical responses observation, enabling characterization performance including design capacity (allowable or maximum compliances) and even monitoring critical deflections at failure. With additional advances, DIC could also be a part of an inplace monitoring network providing real time analysis for structural beams or capture volumetric evaluation of structural beams or systems using two cameras. DIC has great potential to continued advancement and expansion solidifying its ability as an indispensable tool for in civil engineering evaluations.

Acknowledgements. The first author would like to acknowledge the financial support including Michigan Tech Department of Civil \& Environmental Engineering, Michigan Tech Graduate School, King Chavez Parks Future Faculty Fellowship, and USDOT project under the number DTOS59-10-H00001 .

\section{References}

1. W. B. Müller \& B.A. Reeves. "Comparing traffic speed deflectometer and noise-modulated ground penetrating radar data for rapid road pavement investigations". In Ground Penetrating Radar (GPR) 14th International Conference on. IEEE, pp. 502-509. 2012.

2. A. N. Hanna, "Determination of Insitu Material Properties of Asphalt Concrete Pavement Layers from Nondestructive Tests". NCHRP Research Results Digest, vol. 271, 2002.

3. M. Malesa, K. Malowany, U. Tomczak, B. Siwek, M. Kujawińska and A. Siemińska-Lewandowska "Application of 3D digital image correlation in maintenance and process control in industry." Computers in Industry. 2013.

4. S.Ri, T. Numayama, M. Saka, K. Nanbara, and D. Kobayashi. 'Noncontact deflection distribution measurement for large-scale structures by advanced image processing technique". Materials Transactions, vol 53, no 2, pp. 323329,2012 .

5. P.C Hung. and A. S. Voloshin. "In-plane strain measurement by digital image correlation." Journal of the Brazilian Society of Mechanical Sciences and Engineering vol. 25, pp. 215-221, 2003.

6. R.Oats, D. Harris, T. Ahlborn and H. de Melo e Silva. "An Evaluation of Digital Image Correlation as a Structural Damage Assessment and Management Tool". Proceedings of Transportation Research Board 92nd Annual Meeting, TRB. 2013.

7. B. Pan, D. Wu, Y. Xia and A. Asundi. "An active imaging digital image correlation method for deformation measurement insensitive to ambient light." Optics \& Laser Technology vol. 44, pp. 204-209, 2012.

8. I. S. Koltsida, A. K. Tomor and C. A. Booth. "The Use of Digital Image Correlation Technique for Monitoring Masonry Arch Bridges". 7th International Conference on Arch Bridges. pp. 681-690, 2013.

9. B. Pan, K. Qian, H. Xie and A. Asundi. "Two-dimensional digital image correlation for in-plane displacement and strain measurement: A review." Measurement Science and Technology. vol. 20, 2009.

10. A. Hosseini, D. Mostofinejad, and M. Hajialilue-Bonab. "Displacement measurement of bending tests using digital image analysis method." International Journal of Engineering and Technology. vol 4, no.5, pp. 642, 2001. 2012.

11. Correlated Solutions. 2016." The Vic2D System" Non-Contacting Measurement Solutions. Available: http://correlatedsolutions.com/vic-2d/

12. M. Sutton, J. J. Orteu and H. Schreier. Image Correlation for Shape, Motion and Deformation Measurements Basics Concepts, Theory and Applications. New York, USA, Springer. 2009. 
13. M. G., Iskander, S. Sadek and J. Liu. "Soil Structure Interaction in Transportation Synthetic Soils Using Digital Image Correlation". Transportation Research Board Annual Meeting Washington, DC. TRB Session on Recent Advanced in Modeling Techniques in GeoMechanics. 2003.

14. H. Lemmen, R. Alderliesten, R. Benedictus, J. Hofstede and R. Rodi. "The power of Digital Image Correlation for detailed elastic-plastic strain measurements". WSEAS International Conference on Engineering Mechanics, Structures, Engineering Geology. 2008.

15. S. Yoneyama, H. Kikuta, A. Kitagawa, and K. Kitamura. "Lens distortion correction for digital image correlation by measuring rigid body displacement." Optical Engineering. vol 45, no. 2, 2006.

16. B. Pan, L. Yu, and D. Wu. High-accuracy 2D digital image correlation measurements using low-cost imaging lenses: implementation of a generalized compensation method. Measurement Science and Technology, vol. 25, no 2, 2013.

17. S. Yoneyama, A. Kitagawa, S. Iwata, K. Tani and H. Kikuta. "Bridge deflection measurement using digital image correlation." Experimental Techniques. vol. 31, no.1, pp 34-40, 2007.

18. E. Santini-Bell, P. Brogan, P. Lefebvre, J. Peddle, B. R. Brenner and M. Sanayei. Digital Imaging for Bridge Deflection Measurement of a Steel Girder Composite Bridge. Presented at 90th Meeting of the Transportation Research Board. 2011.

19. N. J. McCormick, and J. D. Lord. Practical in-situ applications of DIC for large structures. Yth International Conference on Advances in Experimental Mechanics, Trans Tech Publications. 2010.

20. B. Pan, Z. Lu, and H. Xie. Mean intensity gradient: an effective global parameter for quality assessment of the speckle patterns used in digital image correlation. Optics and Lasers in Engineering, vol. 48, no. 4, pp. 469-477, 2010 .

21. G. Crammond, S.W. Boyd and J.M. Dulieu-Barton. "Speckle pattern quality assessment for digital image correlation". Optics and Lasers in Engineering, vol. 51, no.12, pp. 1368-1378, 2013.

22. R. Cintron and V. Saouma. "Strain Measurements with Digital Image Correlation System with Vic-2D". NEES at University of Colorado, Boulder, 2008.

23. The Imaging Source. "A Theoretical and Practical Introduction to Optics. White Paper". 2010. Available: http://s1.archive.theimagingsource.com/publications/whitepapers-optics/wpbasico pt/5391254a2a9b7d7c23fe039ae3e8865c900a53122838c4e7858e0b1f093d9332/wpbasicopt.en_US.pdf

24. M.Dutton, W. A. Take, and N.A. Hoult. Curvature monitoring of beams using digital image correlation. Journal of Bridge Engineering, vol. 19, no.3, 2013.

25. J. F. Destrebecq, E. Toussaint, and E. Ferrier. "Analysis of Cracks and Deformations in a Full Scale Reinforced Concrete Beam Using a Digital Image Correlation Technique.” Experimental Mechanics. vol. 51, no. 6, pp. 879$890,2010$.

26. E. Jones. 2013. "Improved digital image correlation (DIC)". Available:

http://www.mathworks.com/matlabcentral /flexexchange/43073-improved-digital-image-correlation-dic- 